\title{
ARTIFICIAL SOCIAL ETHICS: SIMULATING CULTURE, CONFLICT, AND COOPERATION
}

\author{
F. LeRon Shults \\ University of Agder and NORCE \\ Center for Modeling Social Systems \\ Universitetsveien 19, SKP Building \\ 4630 Kristiansand, NORWAY \\ leron.shults@uia.no
}

\author{
Wesley J. Wildman \\ Boston University and the \\ Center for Mind and Culture \\ 566 Commonwealth Avenue, Suite M-2 \\ Boston, MA 02215, USA \\ wwildman@bu.edu
}

\begin{abstract}
In recent years advances in computational modeling and social simulation technologies have enabled scientists to identify some of the conditions under which - and the mechanisms by which - conflict and cooperation within and across human cultures are likely to emerge. There are significant ethical concerns surrounding the increased capacity and growing use of such computer tools to guide public policy discussions. The purpose of this paper is to propose and promote an "artificial social ethics" approach to addressing these concerns and illustrate its application in relation to three agent-based models implemented within the Artificial Society Analytics Platform. We conclude with a brief discussion of next steps in future work, and an invitation to join the ethical conversation around the development and deployment of artificial societies designed to address societal challenges resulting from climate change and other crises.
\end{abstract}

Keywords: social ethics, artificial societies, conflict, cooperation

\section{INTRODUCTION}

The use of artificial societies and social simulation to study human behaviors and interactions has increased rapidly in recent years and spread across a wide range of disciplines, including cognitive science, psychology, sociology and the humanities (Sun, 2012; Squazzoni, Jager, \& Edmonds, 2014; Diallo, Wildman, Shults, \& Tolk, 2019). As scholars within this multi-disciplinary scientific community have successfully developed and deployed computational models that illuminate and explain complex psychosocial systems over the last couple of decades, debates over standards, methodologies and appropriate topics for modeling have become relatively stabilized (Hauke, Lorscheid, \& Meyer, 2017). As with any new scientific field experimenting with novel methods, practitioners of computational modeling and simulation (M\&S) should be careful not to overstate the value or overestimate the validity of the analytic and forecasting tools they construct. However, advances in social simulation are increasingly attracting the attention of policy professionals and news media; for example, the title of a recent article in New Scientist magazine read "Predicting the future is now possible with powerful new AI simulations" (Lawton, 2019).

This sort of attention-grabbing headline quite understandably raises a host of ethical questions about the assumptions, purposes, and uses of multi-agent artificial intelligence models, all of which must be transparently addressed and openly discussed as these tools are increasingly applied to the exploration of alternative human futures (Shults \& Wildman, 2019). We accept and acknowledge the challenges involved in this process (Shults, Wildman \& Dignum, 2018), but in this paper we focus on some of the opportunities that social simulation provides for studying and promoting altruistic behaviors and interactions as our species faces existential threats in an increasingly ecologically, economically, and politically complex 
global environment. We think that the multi-disciplinary community of scholars who engage in cognitive and social simulation through the construction of (and experimentation on) artificial societies has a unique and valuable role to play here. M\&S tools, and especially multi-agent artificial intelligence models (MAAI; Lane, 2013), have several properties that commend their use in tackling the task of simulating complex adaptive social and natural systems. These include their capacity

- to encourage the specification of (ethical) assumptions and purposes in a model,

- to inform policy analysis and planning through forecasting scenario experiments,

- to utilize big data as well as diverse data in the verification and validation process, and

- to enable the coupling of human and natural (or social and ecological) systems.

In this paper, we highlight the importance of reflection on social ethical issues surrounding the development and deployment of such artificial societies (section 2) and illustrate this importance in the context of three computational models that simulate social dynamics impacted by moral norms (section 3) using the Artificial Society Analytics Platform. We conclude (section 4) with a brief discussion of next steps in future work, and a call to M\&S practitioners to engage in the increasingly important conversations about the ethical assumptions and implications of modeling moral behaviors and social norms.

\section{ARTIFICIAL SOCIAL ETHICS}

Our interest here is in the social ethical issues surrounding the construction of multi-agent artificial intelligence (MAAI) simulations in the form of "artificial societies." Ethical analysis of MAAI-type artificial societies is not starting from zero as there has been significant reflection on ethical issues connected to the more general field of artificial intelligence (AI), which typically focuses more on the creation of simulated individual agents. We begin by describing some of the frameworks that guide this broader conversation, and then explore their helpfulness and limitations in relation to the conversation we are trying to promote on artificial social ethics.

Ethical debates within artificial intelligence more generally can be divided into two branches (Winfield et. al. 2019). The first branch, commonly referred to as "AI ethics" or "robot ethics," deals with ethical issues surrounding the design and use (or misuse) of AI. For example, how should programmers deploy AI to determine which advertisements are displayed to a social-media user, how much an airline ticket should cost, and when stocks should be bought and sold or currencies traded? What algorithms should programmers use to determine relative life value when guiding an autonomous vehicle's behavior in the face of a unusual driving situations that require a decision about whom to hit if the vehicle cannot stop quickly enough (e.g., an elderly woman or a little boy). Cross-cultural experiments show that humans make quite different kinds of moral assessments in such situations; this makes sense given the fact that moral learning occurs as individuals interact in social groups with varying cultural norms, but it complicates debates over the "right" way to program autonomous vehicles to act in such situations (Awad et al., 2018; Kleiman-Weiner, Saxe, \& Tenenbaum, 2017). Not surprisingly, worries about the ethical use of AI commonly arise in areas where we humans feel especially ambivalent about the morality of our own actions. This is perhaps most obvious when AI is developed for activities related to "making war" (the military use of AI that could produce "killer robots": Lin, Abney, \& Bekey, 2014; Noorman \& Johnson, 2014) or "making love" (the commercial use of AI that is already producing "sex robots": Richardson, 2016; Danaher \& McArthur, 2017).

The second branch, typically referred to as "ethical AI," or sometimes more broadly as "machine ethics" (Anderson \& Anderson, 2011), explores questions about whether it is possible - and, if so, what it would mean - for AI to behave "ethically." Here scholars debate the potential value and risks of creating ethical machines (Cave, Nyrup, Vold, \& Weller, 2018). In the autonomous vehicle example above, this is equivalent to regarding the vehicle as literally morally autonomous, a moral machine deciding how to 
behave, including how to solve real-life versions of the philosopher's infamous "trolley problem." This kind of moral machine could be programmed to learn ethics by interacting with an environment and receiving feedback, thereby making its own decisions, rather than being controlled by an exhaustive suite of algorithms conveying moral decisions made by human designers and programmers.

These intertwined branches are of interest to both computer engineers and philosophers, conversations between whom are increasingly important. "Philosophers are interested in the outcomes of practical machine ethics not least because - if successful - they lend urgency to the moral questions around ethical machines in society. Equally, engineers engaged in designing ethical machines need philosophers to advise on the definition of appropriate ethical rules and values for these machines" (Winfield et al., 2019, p. 510). And the very same experts need each other as they invest in understanding the ethics of applying AI algorithms in real-world situations.

In both branches of this academic and wider public discussion, the focus is usually on the construction (or use) of singular agents who are more or less artificially intelligent and (potentially) artificially moral or individual algorithms. This is not all that surprising since the major ethical schools of thought in western philosophy have traditionally focused primarily on individual human agents. Deontologists argue about the rules individual agents should follow, consequentialists about the utility functions individual agents should optimize, and virtue ethicists about the character traits dispositions individual agents should cultivate. Ethical computational architectures representing each of these schools have been implemented within individual artificial agents (Allen, Varner, \& Zinser, 2000; M. Anderson, Anderson, \& Armen, 2005; Grau, 2011; Powers, 2006). None of these philosophical schools ignore the communal dimensions of human ethics, but they do not typically emphasize the way in which group dynamics, social contexts, cultural norms, and familial and other network relations play a role in motivating and justifying moral behaviors. In other words, they are more focused on individual ethics than on social ethics.

The development of MAAI models and social simulations of morally salient behaviors (such as conflict and cooperation) within and across groups has not received as much attention among ethicists or the general public, perhaps because advances in this field are more recent and the phenomena they simulate are more complex or less intuitively accessible. As this field continues to grow, however, it is garnering more attention (e.g., Lawton, 2019). We believe that fostering conversations about artificial social ethics will become increasingly important as our species faces new social and economic challenges at local, regional, and global levels. These conversations will be relevant for the development of both "ethical MAAI" (artificial societies with realistic moral agents and interactions) and "machine social ethics" (the ethical application of the scientific and policy-relevant findings of social simulation experiments).

Scholars debate when (or whether) we will reach the "singularity" at which point AI becomes as intelligent as (or more intelligent than) human beings. A great deal hangs on the meaning of "intelligence." Questions about the eventual moral superiority of machines are perhaps even more complicated because there is even less consensus on what it means to be "moral." One popular taxonomy of moral machines defines "explicit ethical agents" as those that would be capable of representing ethics explicitly and "then operate effectively on the basis of this knowledge" (Moor, 2006, p. 20). Some computer scientists argue that many AI agent architectures already qualify as "explicit ethical agents," at least in a limited sense, insofar as they "have either learned or defined ethical rules and the cognitive machinery to take those rules into account when deciding how to act in a given situation..." (Winfield et al., 2019, p. 511). One recent machine-reasoning architecture for robot AI utilizes a simulation-based internal model that enables proactive, transparent, and verifiable ethical reasoning (Bremner et al., 2019). We are not yet at the "moral" singularity where individual AI can make explicit and justify its ethical reasoning, but we may be getting closer.

What about moral artificial societies? How close are we to constructing computational social simulations that mimic the ethical (or unethical) behaviors and patterns of real-world societies? Although comprehensive "digital twins" of human societies do not (yet) exist, state-of-the-art models do have agents 
(and clusters of agents) capable of representing "moral knowledge" and operating effectively on the basis of this knowledge. Several models are capable of simulating the emergence of ethically salient macro-level societal phenomena from micro-level "moral" interactions, including cultural norms (Conte, Andrighetto, \& Campennì, 2014; Elsenbroich \& Gilbert, 2014). Our research teams have developed several computational models that simulate behaviors and interactions that are morally charged, such as in-group reactions to threats (Shults, Lane, Diallo, et al., 2018), the mutual escalation of intergroup conflict (Shults, Gore, Wildman, et al., 2018), and the reduction of religious beliefs and affiliation in secularizing contexts (Gore, Lemos, Shults, \& Wildman, 2018). Below we will explore some other models that attempt to simulate the mechanisms driving human moral interactions well enough to allow us to predict (and prevent or promote) the morally potent issue of the emergence of conflict and cooperation in real world societies.

Elsewhere we have proposed a meta-ethical framework for discussing the development and deployment of artificial societies and social simulations (Shults \& Wildman, 2019; Shults, Wildman, \& Dignum, 2018). This is analogous to what in the AI literature is sometimes called "machine metaethics" (Anderson, 2011), but the sort of reflection on artificial social ethics that we are proposing is more explicitly focused on the way in which cultural norms, social networks, and other environmental parameters shape moral behaviors and interactions, and how that shaping should occur.

The first aspect of this meta-ethical framework is philosophical. This is the most abstract dimension, and invites modelers to make explicit their definitions of "the good" and "the right," and the relation between them. This means being clear about the sense in which one is following a consequentialist, deontological, virtue, or some other approach to ethics. We are suggesting not that all modelers should take the same approach, only that we should be as clear as possible about the ethical assumptions shaping modeling activities. For our part, we recommend a broadly pragmatic consequentialist framing of ethical issues, primarily because both deontological and virtue approaches (whatever their benefits) have traditionally been framed in relation to transcendental ideals or exemplars of the sort that play a role in the ethical systems of religious in-groups. Consequentialist approaches can also be framed in this way, but we think that this philosophical approach is more conducive to the sort of trans-communal discourse about moral goods that will be needed to respond to the global challenges of the current era.

The second aspect of our framework is scientific, and draws specifically on the bio-cultural sciences that bear on the evolution of our moral instincts, emotions, and reasoning. Here our recommendation is that modelers attempting to construct realistic cognitive architectures and social networks by taking seriously the insights from disciplines such as evolutionary biology and moral psychology about the evolved tendency of members of our species to implicitly accept and follow the cultural and religious norms of the in-groups in which we are raised. While it makes sense that this tendency would have been naturally selected in contexts where the cohesion of small-scale homogeneous societies was necessary for survival, in contemporary large-scale societies, characterized by diverse and dense populations, such a tendency can all too quickly flip into anxiety about and aggression toward out-group members, especially under stressful conditions (Wildman, 2009; Shults, 2018).

Finally, the practical aspect of the meta-ethical framework spells out the pragmatic implications of the first two aspects. Surfacing and clarifying the philosophical assumptions that shape our construction of artificial societies and our criteria for evaluating the success of simulation experiments can help us avoid moral confusion when talking to one another about the social ethical implications of our efforts as M\&S practitioners. The incorporation of scientific insights about the evolved mechanisms that shape human ethical behavior within and among groups can help us avoid moral evasion of the sort that appeals to the hidden supernatural agents of a particular religious coalition (e.g., "the ancestors require it of us"). Learning how to live together on the same planet, while limiting conflict and promoting cooperation beyond parochial and national interests as we respond to the impacts of climate change and other global threats, will require us to take responsibility for our own actions as individuals and as groups. In the next section, we briefly 
illustrate the relevance and importance of this sort of artificial social ethical reflection in the context of a concise presentation of three computational models recently constructed by our research teams.

\section{SIMULATING CONFLICT AND COOPERATION ON THE ARTIFICIAL SOCIETY ANALYTICS PLATFORM}

Each of the three models we briefly discuss here was implemented on one or another version of the previously developed Artificial Society Analytics Platform or ASAP (Shults, Wildman, Diallo, PugaGonzalez, \& Voas, 2018). ASAP was designed to handle a variety of specialized research questions dealing with the evaluation of scientific hypotheses about healthy social equilibria within urban areas in the developed western world. Agents in ASAP are distributed in different neighborhoods and linked to job locations. They meet in a variety of networks, influence one another's worldviews, get educated, seek employment, look for compatible marriage partners and reproduce, age, and die. They can shift worldviews and join (or leave) worldview clubs. Agents also have the sort of demographic variables that would be minimally required to simulate any interesting and ethically salient social dynamics, such as age, gender, income level, membership in a minority or majority group, etc.

In addition to demographic variables, agents also have variables designed to help us simulate various types of integration within heterogenous societies. We think this is particularly important if the goal is simulating solutions for resolving conflict and promoting cooperation among groups with different norms and values in politically complex societies and as climate change turns up the (social) heat by inducing resource scarcity and forcing migration. Several sociological theories were taken into account in framing the integration variables, but the computational architecture was strongly informed by the personal involvement and research of sociologist and demographer David Voas (see, e.g., Voas \& Bruce, 2014). These variables help us capture the difference between three hypothesized dimensions or types of integration: 1) structural integration, which is characterized by equality of opportunity in education, employment, housing, civil rights, and civic participation across groups; 2) social integration, which is defined as interaction between members of different groups in ways that range from superficial (brief impersonal or commercial transactions) to deeply personal (close friendships and intimate relationships); and 3) cultural integration, which involves the sharing of norms, values, worldviews, and cultural capital across groups (PugaGonzalez, Wildman, Diallo, \& Shults, 2019).

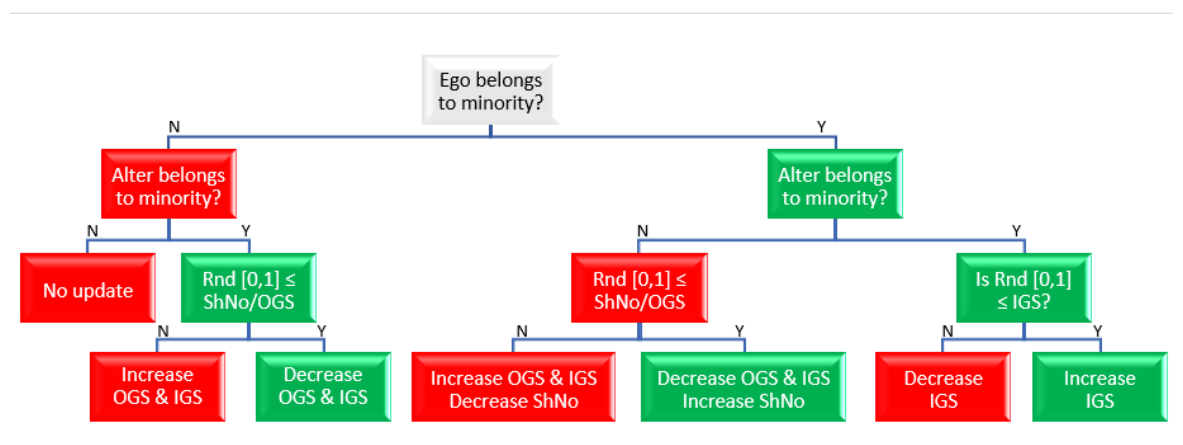

Figure 1: Dyadic neighborhood interactions in ASAP. Ego=agent possibly changing; alter=ego's interaction partner; Rnd [0,1]=random number between 0 and 1; IGS=ingroup support; OGS=outgroup suspicion; $\mathrm{ShNo}=$ shared norms.

The agents in ASAP also have personality variables, which impact moral judgments and are distributed in the artificial population in ways that reflect real societies. We adopted the HEXACO framework (Aghababaei, 2014) because it extends the Big Five framework (Gebauer et al., 2014) by adding Honesty/Humility, which is an important factor in some interpersonal interactions and moral appraisals. Incorporating personality helps us enrich the representation of relevant social dynamics, such as shifts in 
agent worldviews, switching in worldview club affiliation, and morally laden appraisal such as outgroup suspicion, ingroup support, and shared norms. For example, religiously affiliated individuals tend to be higher in conscientiousness and lower in openness than the general population. Each week an agent engages in a variety of interactions, leading to changes in agent variables depending on the qualities of the interaction. Figure 1 portrays the decision tree for an ego agent interacting with an alter agent in ego's neighborhood as the ego agent evaluates ingroup support (the degree to which ego relies on social support from ego's ingroup), outgroup suspicion (the degree to which ego is wary of people outside ego's ingroup), and shared norms (the degree to which the entire population shares moral norms).

The following three models are among those we have built on ASAP. Each attempts to simulate ethically salient behaviors and social interactions of the sort that can promote cooperation or prevent conflict as our species responds to climate change and other complex ecological challenges. The aim of each model is to explain how a real-world causal process works rather than to evaluate strategic policy options or make predictions, but these models could be brought into the applied domain as suitable policy challenges and corresponding datasets become available.

First, the CRED model examines the role of credibility-enhancing displays (CREDs) in shaping the prevalence of supernatural beliefs in a population. CREDs are critical factors in the spread of moral norms and supernatural beliefs, often against pressure to avoid costly commitments (on religion, see Langston, 2020; on public goods, see Kraft-Todd, Bollinger, et al., 2018). Traditionally, supernatural worldviews have been critical factors in generating and sustaining moral norms for large numbers of human beings, so the declining plausibility of such worldviews in secularizing societies raises pointed questions about social stability as human beings construct other ways of grounding their moral behavior and reasoning. To develop the CRED model, we added variables to ASAP that allow individuals to hold religious or secular worldviews (from secular to religious on a continuum [0,1]), and to join or leave worldview clubs (religious or secular). Worldview clubs are membership organizations that exist to support people having specific types of worldview and to advance those worldviews. Each club has a charismatic leader, following wellestablished research from sociology of religion (e.g. Weber, 1978). When an agent belongs to a worldview club, the agent's personal worldview variable tends to match the worldview of the club. Further, we also endowed agents with personality characteristics beyond the HEXACO factors: charisma, susceptibility, frustration and hypocrisy tolerance. HEXACO personality variables affect the agents' initial worldview, their tendency to join and leave clubs, and the consistency and magnitude with which they display CREDs; the other personality variables are especially important for interpersonal interactions, including how persuasive each agent finds another agent to be. Both worldview and club membership are impacted by the intensity and consistency of CREDs in the environment. The CRED model is still in development but it promises to shed light on the conditions under which supernatural forms of religion fade within an artificial society designed to simulate contexts such as those populated by inCREDulous Scandinavians (PugaGonzalez, Wildman, McCaffree, Cragun, \& Shults, 2019) and investigate social stability under such conditions.

Second, we adapted ASAP to investigate the conditions under which prosocial (or altruistic) attitudes and tendencies increase or decrease in western populations. The computational architecture of this Prosociality model was based on a structural equation model derived from longitudinal statistical analysis of World Values Survey data (Gore, Zuckerman, Galen, Pollack, \& Shults, 2019). Experimental simulation results from this model showed that affiliation with supernaturalist worldview groups (e.g., churches or mosques) is associated with increased "active" prosociality but this is largely due to greater opportunities for in-group altruism (e.g., donating, volunteering). Discounting "tribal" (in-group) prosocial actions, individuals with a naturalist worldview are actively prosocial at the same rate as individuals with a supernaturalist worldview. When it comes to "passive" prosociality (trust, tolerance), on the other hand, individuals affiliated with supernaturalist worldview groups are not as altruistic as unaffiliated individuals with a naturalist worldview. Our modeling suggests that this is due to the homophily characteristics of supernaturalist worldview clubs, whose social networks tend to be homogeneous, which reduces exposure 
to outgroup members and increases the likelihood of interacting with others who have high outgroup suspicion (fellow affiliated believers). Simulation experiments have also provided insights into changes in belief and affiliation that occur over time. For example, individuals with a supernaturalist worldview who move from being affiliated with a supernatural worldview club to being unaffiliated exhibit trust of others at a higher rate than individuals with a supernaturalist worldview who move from being unaffiliated to being affiliated with a supernaturalist worldview club. Those who transition from belief to nonbelief (or toward naturalist worldviews) are largely made up of the unaffiliated who also have lower outgroup suspicion and more heterogeneous networks that feature belief-eroding encounters.

Third, we used ASAP to build a Cultural Norms model that tests the effects of culturally varied moral norms (Diallo, Shults, \& Wildman, under review). This is a vital consideration as migration due to political instability and climate change places people with quite different culturally borne moral norms in close proximity to one another, producing both the potential for sharp social conflict and the opportunity for cooperation in the form of cross-cultural understanding. For example, in some cultures, unmediated interactions between adult males and female children is extremely rare, while in other cultures it is more common. The Cultural Norms model allows us to compare social behavior in the two cases, taking account of the coexistence of these norms in a cultural context that contains majority and minority populations. When the model does not allow adult males in the majority to interact directly with female children in the minority, those children are only influenced by males in the minority and females and children in both groups. As a result, the overwhelming presence of males of the majority group does not overtake the interaction space, which means that there is potential for localized and isolated effects such as alienation for teenagers and integration for adults of the same group. The Cultural Norms model decisively demonstrates that we must take account of culturally borne social norms when assessing how integration of a minority migrant community might occur within a majority western society.

These three implementations of the ASAP system study different aspects of the social dimension of morality. Collectively, these models illustrate the value of introducing artificial social ethics into broader discussions of AI and ethics, as we struggle to develop new responses to contemporary societal challenges. The results of the simulation experiments on these models demonstrate the importance of going beyond treating AI agents merely as autonomous individual moral agents. We also need to take account of factors such as the culturally specific nature of social moral norms, the personality-based character of moral learning, and the complexities of moral behavior and reasoning.

\section{CONCLUSION}

In this paper we have argued that extending AI ethics to incorporate and even emphasize artificial social ethics is critical for ensuring that computer simulations become relevant and widely accepted methods for studying and responding to the challenges facing contemporary human societies. We believe there is a profound opportunity here to unite what philosophers know about social ethics with what simulation engineers know about artificial societies, thereby providing powerful analytical tools that are directly relevant for policy debates.

To realize that potential, however, much work is needed. Fundamentally, we need to study all of the many different aspects of morally complex social realities, including norm emergence, polarization, populism, sustainability, and the social impacts of global threats such as climate change. In short, we need to investigate the moral landscape of the Anthropocene. The latter term is increasingly used among climate scientists and other scholars to refer to the current geological era in which human activity has become the dominant global influence on the climate and the environment (Davies, 2016; McNeill \& Engelke, 2016; Shults, 2015; Steffen, Broadgate, Deutsch, Gaffney, \& Ludwig, 2015). The growing consensus among scholars who study these issues is that we are running out of time to address them; unless we can focus our attention on the problem and come up with new solutions our species faces extinction or mass suffering within the next few generations (Klein, 2015; Kolbert, 2014). In recent years the United Nations and other 
international organizations have increasingly invested energy into facilitating dialogue, motivating action, and generating policies in ways that attempt to arrest climate change while also preparing for the effects of climate change on conflicts within and between groups as resources become scarce in some times and places and migration becomes more common.

While climate scientists labor to investigate alternative climate scenarios (for example, see Steffen et al., 2018; Rolnick et al., 2019), artificial social ethics is needed to address the resulting social impacts. Stakeholders, policy professionals, social scientists, philosophers, and computer scientists can contribute to a deeper understanding of the conditions under which - and the mechanisms by which - conflict and cooperation within and across human cultures are likely to emerge in the Anthropocene, shedding light on relevant tipping points in a way that could inform interventions capable of maximizing cooperation and minimizing conflict. Ultimately, this will require computational architectures that couple climate models of the sort developed by natural scientists with conflict and cooperation models of the sort developed by social scientists. Attempts at such a linkage are very recent and very few, and typically use relatively simple simulated agents (e.g., Beckage et al., 2018; Bury, Bauch, \& Anand, 2019). Our research teams are working toward the development of coupled (socio-ecological) models that involve more cognitively realistic agents and more sociologically realistic networks in order to provide a more fully developed artificial social ethics responsive to the challenges of Anthropocene. In this context, we have focused on some of the initial steps toward this goal. We are hopeful that the M\&S community will increasingly bend its efforts in this direction in order to improve the prospects of rational policy deliberation in the face of an uncertain and challenging future for our species.

\section{ACKNOWLEDGMENTS}

The authors are grateful for conversations around these issues with the team members of the Modeling Religion in Norway Project (funded by The Research Council of Norway, grant \#250449) and the Modeling Religion Project (funded by the John Templeton Foundation, grant \#43288).

\section{REFERENCES}

Aghababaei, N. (2014). God, the good life, and HEXACO: The relations among religion, subjective wellbeing and personality. Mental Health, Religion \& Culture, 17(3), 284-290. https://doi.org/10.1080/13674676.2013.797956

Allen, C., Varner, G., \& Zinser, J. (2000). Prolegomena to any future artificial moral agent. Journal of Experimental \& Theoretical Artificial Intelligence, 12(3), 251-261.

Anderson, M., Anderson, S., \& Armen, C. (2005). Towards machine ethics: Implementing two action-based ethical theories. Proceedings of the AAAI 2005 Fall Symposium on Machine Ethics, 1-7.

Anderson, M., \& Anderson, S. L. (2011). Machine ethics. Cambridge: Cambridge University Press.

Anderson, S. L. (2011). Machine Metaethics. In Anderson \& Anderson, eds., Machine Ethics (pp. 21-27). Cambridge: Cambridge University Press.

Awad, E., Dsouza, S., Kim, R., Schulz, J., Henrich, J., Shariff, A., ... Rahwan, I. (2018). The moral machine experiment. Nature, 563(7729), 59.

Beckage, B., Gross, L. J., Lacasse, K., Carr, E., Metcalf, S. S., Winter, J. M., ... Zia, A. (2018). Linking models of human behaviour and climate alters projected climate change. Nature Climate Change, 8(1), 79.

Bremner, P., Dennis, L. A., Fisher, M., \& Winfield, A. F. (2019). On Proactive, Transparent, and Verifiable Ethical Reasoning for Robots. Proceedings of the IEEE, 107(3), 541-561. 
Bury, T. M., Bauch, C. T., \& Anand, M. (2019). Charting pathways to climate change mitigation in a coupled socio-climate model. PLoS Computational Biology, 15(6), e1007000.

Conte, R., Andrighetto, G., \& Campennì, M. (2014). Minding norms: Mechanisms and dynamics of social order in agent societies. Oxford: Oxford University Press.

Danaher, J., \& McArthur, N. (2017). Robot Sex: Social and Ethical Implications. Cambridge: MIT Press.

Davies, J. (2016). The Birth of the Anthropocene. Oakland, California: University of California Press.

Diallo, S., Wildman, W. J., Shults, F. L., \& Tolk, A. (Eds.). (2019). Human Simulation: Perspectives, Insights, and Applications. Berlin: Springer.

Diallo, S. Y., Shults, F. L., \& Wildman, W. J. (under review). Minding Morality: Ethical Artificial Societies for Public Policy Modeling.

Elsenbroich, C., \& Gilbert, N. (2014). Modelling Norms. Dordrecht: Springer Netherlands.

Gebauer, J. E., Bleidorn, W., Gosling, S. D., Rentfrow, P. J., Lamb, M. E., \& Potter, J. (2014). CrossCultural Variations in Big Five Relationships With Religiosity: A Sociocultural Motives Perspective. Journal of Personality and Social Psychology, 107(6), 1064-1091. https://doi.org/10.1037/a0037683

Gore, R., Lemos, C., Shults, F. L., \& Wildman, W. J. (2018). Forecasting changes in religiosity and existential security with an agent-based model. Journal of Artificial Societies and Social Simulation, 21, 1-31.

Gore, R., Zuckerman, P., Galen, L. W., Pollack, D., \& Shults, L. (2019). "Good Without God? Connecting Religiosity, Affiliation And Pro-sociality Using World Values Survey Data And Agent-based Simulation." SocArXiv Papers. https://osf.io/preprints/socarxiv/jnpe9/

Grau, C. (2011). There is no 'I'in 'Robot': Robots and utilitarianism. Machine Ethics, 451-63.

Hauke, J., Lorscheid, I., \& Meyer, M. (2017). Recent development of social simulation as reflected in JASSS between 2008 and 2014: A citation and co-citation analysis. JASSS, 20(1), 1-21. https://doi.org/10.18564/jasss.3238

Kleiman-Weiner, M., Saxe, R., \& Tenenbaum, J. B. (2017). Learning a commonsense moral theory. Cognition, 167, 107-123.

Klein, N. (2015). This Changes Everything: Capitalism vs. The Climate. New York: Simon \& Schuster.

Kolbert, E. (2014). The sixth extinction: An unnatural history. New York: Henry Holt and Co.

Kraft-Todd, G.T., Bollinger, B., Gillingham, K., Lamp, S., \& Rand, D. G. (2018). Credibility-enhancing displays promote the provision of non-normative public goods. Nature 563, 245-248.

Lane, J. (2013). Method, theory, and multi-agent artificial intelligence: Creating computer models of complex social interactions. Journal for the Cognitive Science of Religion, 1(2), 161-180.

Langston, J. 92020). Predicting age of atheism: credibility enhancing displays and religious importance, choice, and conflict in family of upbringing. Religion, Brain \& Behavior 10(1), 49-67.

Lawton, G. (2019, October 5). Predicting the future is now possible with powerful new AI simulations. New Scientist, 38-41.

Lin, P., Abney, K., \& Bekey, G. A. (2014). Robot ethics: The ethical and social implications of robotics. Cambridge: The MIT Press.

McNeill, J. R., \& Engelke, P. (2016). The Great Acceleration: An Environmental History of the Anthropocene since 1945. Cambridge: Belknap Press. 
Moor, J. H. (2006). The nature, importance, and difficulty of machine ethics. IEEE Intelligent Systems, 21(4), 18-21.

Noorman, M., \& Johnson, D. G. (2014). Negotiating autonomy and responsibility in military robots. Ethics and Information Technology, 16(1), 51-62.

Powers, T. M. (2006). Prospects for a Kantian machine. IEEE Intelligent Systems, 21(4), 46-51.

Puga-Gonzalez, I., Wildman, W. J., Diallo, S. Y., \& Shults, F. L. (2019). Minority integration in a western city: An agent-based modeling approach. In Human Simulation: Perspectives, Insights, and Applications. Switzerland: Springer Nature.

Puga-Gonzalez, I., Wildman, W. J., McCaffree, K., Cragun, R., \& Shults, F. L. (2019). InCREDulity in Artificial Societies. Proceedings of the Social Simulation Conference.

Richardson, K. (2016). Sex robot matters: Slavery, the prostituted, and the rights of machines. IEEE Technology and Society Magazine, 35(2), 46-53.

Shults, F. L. (2015). How to Survive the Anthropocene: Adaptive Atheism and the Evolution of Homo deiparensis. Religions, 6(2), 1-18. https://doi.org/10.3390/rel6020724

Shults, F. L. (2018). Practicing Safe Sects: Religious Reproduction in Scientific and Philosophical Perspective. Leiden: Brill Academic.

Shults, F. L., Gore, R., Wildman, W. J., Lynch, C., Lane, J. E., \& Toft, M. (2018). A Generative Model of the Mutual Escalation of Anxiety Between Religious Groups. Journal of Artificial Societies and Social Simulation, 21(4), DOI: 10.18564/jasss.3840.

Shults, F. L., Lane, J. E., Diallo, S., Lynch, C., Wildman, W. J., \& Gore, R. (2018). Modeling Terror Management Theory: Computer Simulations of the Impact of Mortality Salience on Religiosity. Religion, Brain \& Behavior, 8(1), 77-100.

Shults, F. L., \& Wildman, W. J. (2019). Ethics, computer simulation, and the future of humanity. In S. Y. Diallo, W. J. Wildman, F. L. Shults, \& A. Tolk (Eds.), Human Simulation: Perspectives, Insights and Applications (pp. 21-40). Berlin: Springer.

Shults, F. L., Wildman, W. J., Diallo, S., Puga-Gonzalez, I., \& Voas, D. (2018). The Artificial Society Analytics Platform. Proceedings of the 2018 Social Simulation Conference, 1-12.

Shults, F. L., Wildman, W. J., \& Dignum, V. (2018). The ethics of computer modeling and simulation. Proceedings of the Winter Simulation Conference (WSC), 4069-4083. IEEE.

Squazzoni, F., Jager, W., \& Edmonds, B. (2014). Social Simulation in the Social Sciences. Social Science Computer Review, 32(3), 279-294. https://doi.org/10.1177/0894439313512975

Steffen, W., Broadgate, W., Deutsch, L., Gaffney, O., \& Ludwig, C. (2015). The trajectory of the Anthropocene: The Great Acceleration. The Anthropocene Review, 2(1), 81-98. https://doi.org/10.1177/2053019614564785

Sun, R. (2012). Grounding Social Sciences in Cognitive Sciences. Cambridge: MIT Press.

Voas, D., \& Bruce, S. (2014). Secularization in Europe: An Analysis of Inter-Generational Religious Change. In W. Arts \& L. Halman (Eds.), Value Contrasts and Consensus in Present-Day Europe. Leiden: Brill.

Voas, D., \& Fleischmann, F. (2012). Islam Moves West: Religious Change in the First and Second Generations. Annual Review of Sociology, 38, 525-545. https://doi.org/10.1146/annurev-soc071811-145455 
Weber, M. (1978). Economy and Society: An Outline of Interpretive Sociology. G. Roth \& C. Wittich (Eds.). Berkeley: University of California Press.

Wildman, W. J. (2009). Science and Religious Anthropology: A Spiritually Evocative Naturalist Interpretation of Human Life. London: Ashgate.

Winfield, A. F., Michael, K., Pitt, J., \& Evers, V. (2019). Machine ethics: The design and governance of ethical AI and autonomous systems. Proceedings of the IEEE, 107(3), 509-517.

F. LERON SHULTS is Professor at the Institute for Global Development and Social Planning at the University of Agder and Scientific Director of the NORCE Center for Modeling Social Systems in Kristiansand, Norway. His email address is leron.shults@uia.no.

WESLEY J. WILDMAN is Professor of Philosophy, Theology, and Ethics at Boston University and Executive Director of the Center for Mind and Culture in Boston, USA. His email address is wwildman@bu.edu. 